\title{
Special Issue: Cell Mechanics and Signaling: From Micro to Nano
}

Mechanical forces play an increasingly recognized role in guiding cell function. Cell-generated forces allow active traversal of both matrix and other cells in the extracellular space, an essential element of tissue development and key to multiple pathologies. Moreover, the response of the environment to these forces conveys information back to the cell, casting mechanics as a cell signaling factor. Determining the factors that guide cell mechanics is a major contemporary challenge across multiple disciplines, for which bioengineering provides a particularly powerful set of concepts and methods. Cells use a collection of physical and chemical cues to produce motion, in many ways similar to how automobiles depend on mechanical, electrical, and hydraulic elements for proper function, and full understanding of either system requires a systems-level integration of these components. In the case of cells, these systems include molecular receptors that sense the extracellular environment, biochemical processing of the various inputs through intricate cell signaling network, and finally transduction of biochemical signals into coordinated mechanical forces. Since these events are orchestrated in a precise spatiotemporal fashion, there is a need to study biochemical and biomechanical processes in cells at both spatially- and temporally-resolved details. Contemporary engineering approaches are well suited for integrating multiple conceptual components and embracing spatially complex processes, particularly at micrometer and nanometer scales between those of tissues and individual protein molecules. The papers in this Special Issue provide a collective snapshot of contemporary progress in this field, highlighting specific, underlying areas of research.

\section{NUMERICAL MODELING}

Computational models are a key engineering approach for investigating complex, physiologically relevant systems. The articles by $\mathrm{Ma}$ et al. $^{3}$ and $\mathrm{Su}$ and Schmid-Schonbein ${ }^{6}$ provide elegant demonstrations of this approach. At sub-cellular scales, Welf and $\mathrm{Haugh}^{8}$ provide a new framework for applying chemical regulatory motifs to local dynamics of cell protrusion.

\section{MATERIALS}

Several materials, particularly polyacrylamide and polydimethylsiloxane, have been developed in the context of cell biomechanics, allowing the study of cell response to materials of more physiological stiffness than traditional cell-culture plastic or glass. In this Issue, Mann and Leckband ${ }^{4}$ continue the development of these materials for longer-term measurement of cellular forces, allowing analysis of these forces over the course of cell differentiation. Zhou and Petroll ${ }^{9}$ examine cell behavior within an advanced, nested collagen matrix.

\section{CELLULAR BIOMECHANICS}

Understanding the regulatory mechanisms of force generation and rigidity sensing by cells is a major contemporary challenge. Moving beyond the analysis of individual cells, the works in this Issue by Liu and Chen, ${ }^{2}$ Saunders and Hammer, ${ }^{5}$ and Califano and Reinhart-King ${ }^{1}$ present new advances into this field, focusing on the roles of cell-generated forces in coordinating heterotypic and homotypic cell-cell interactions. In addition, Mann and Leckband ${ }^{4}$ provide a new glimpse into the dynamics of forces over the course of cell differentiation.

\section{CELL SIGNALING AND BIOPHYSICS}

Proteins, including those with interface and intracellular signaling roles, are the links between mechanics and cell function. In this issue, Welf and Haugh, ${ }^{8}$ Zhou and Petroll, ${ }^{9}$ and Tsai and $\mathrm{Kam}^{7}$ provide elegant, engineering-based studies into the roles of specific proteins, and the biophysical presentation of such molecules, in modulating cell function.

We look forward to the bright future of bioengineering research that utilizes interdisciplinary concepts and improved methods for analyzing spatial complexity of cells, and hence continues to reveal new rules explaining integration of mechanics and signaling at cellular and molecular levels. We also celebrate the first two years of Cellular and Molecular Bioengineering, an important venue for this increasingly interdisciplinary field. We thank the editors David Odde and Edward Guo as well as Springer for their vision and commitment in this field. 


\section{REFERENCES}

${ }^{1}$ Califano, J. P., and C. A. Reinhart-King. Substrate stiffness and cell area predict cellular traction stresses in single cells and cells in contact.

${ }^{2}$ Liu, Z., and C. S. Chen. Mechanical forces in endothelial cells during firm adhesion and early transmigration of human monocytes.

${ }^{3} \mathrm{Ma}$, Y., J. Wang, et al. Application of population dynamics to study heterotypic cell aggregations in the near-wall region of a shear flow.

${ }^{4}$ Mann, C., and D. E. Leckband. Measuring traction forces in long-term cell cultures.

${ }^{5}$ Saunders, R. L., and D. A. Hammer. Assembly of human umbilical vein endothelial cells on compliant hydrogels.

${ }^{6} \mathrm{Su}, \mathrm{S}$. S., and G. W. Schmid-Schonbein. Internalization of formyl peptide receptor in leukocytes subject to fluid stresses.

${ }^{7}$ Tsai, J., and L. C. Kam. Lateral mobility of E-cadherin enhances Rac1 response in epithelial cells.
${ }^{8}$ Welf, E. S., and J. M. Haugh. Stochastic dynamics of membrane protrusion mediated by the DOCK180/Rac pathway in migrating cells.

${ }^{9}$ Zhou, C., and W. M. Petroll. Rho kinase regulation of fibroblast migratory mechanics in fibrillar collagen matrices.

LANCE C. KAM

Department of Biomedical Engineering

Columbia University

Electronic mail:1k2141@columbia.edu

PARTHA RoY

Department of Bioengineering and Pathology

University of Pittsburgh

Electronic mail: par19@pitt.edu 